\title{
N9 94292316
}

\section{The evolution equation for the flame surface density in turbulent premixed combustion}

\author{
By Arnaud Trouvé
}

\section{Motivation and objectives}

The mean reaction rate in flamelet models for turbulent premixed combustion depends on two basic quantities: a mean chemical rate, called the flamelet speed, and the flame surface density (Section 1.1). Our previous work had been primarily focused on the problem of the structure and topology of turbulent premixed flames, and it was then determined that the flamelet speed, when space-averaged, is only weakly sensitive to the turbulent flow field (Haworth \& Poinsot 1992, Rutland \& Trouvé 1991, Trouvé 1991). Consequently, the flame surface density is the key quantity that conveys most of the effects of the turbulence on the rate of energy release. In flamelet models, this quantity is obtained via a modeled transport equation called the $\Sigma$-equation. Past theoretical work has produced a rigorous approach that leads to an exact but unclosed formulation for the turbulent $\Sigma$-equation (Section 1.2). In the exact $\Sigma$-equation, it appears that the dynamical properties of the flame surface density are determined by a single parameter, namely the turbulent flame stretch. Unfortunately, the turbulent flame stretch as well as the flame surface density is not available from experiments, and, in the absence of experimental data, little is known on the validity of the closure assumptions used in current flamelet models.

Direct Numerical Simulation (DNS) is the alternative approach to get basic information on these fundamental quantities. In the present work, three-dimensional DNS of premixed flames in isotropic turbulent flow is used to estimate the different terms appearing in the $\Sigma$-equation (Section 1.3). A new methodology is proposed to provide the source and sink terms for the flame surface density, resolved both temporally and spatially throughout the turbulent flame brush (Section 2.1). Using this methodology, our objective is to extract the turbulent flame stretch from the DNS data base and then perform extensive comparisons with flamelet models (Section 2.3). Thanks to the detailed information produced by the DNS-based analysis, it is expected that this type of comparison will not only underscore the shortcomings of current models, but also suggest ways to improve them.

\subsection{The flamelet approach for turbulent premixed combustion}

Flamelet models constitute one of the most common approaches for turbulent premixed combustion (Bray 1980, Williams 1985, Peters 1986). Such models are very attractive as they conveniently separate the combustion problem from the analysis of the turbulent flow field. These models are based on the "flame sheet" assumption, which requires chemical reaction to occur at fast time scales and short length scales relative to the turbulence. In this situation, the flame is confined to relatively thin layers within the turbulent flow field, and the topology of the 
reaction zone corresponds to a surface burning mode. Experimental, theoretical, and numerical evidence suggest that the domain of validity of the flamelet picture is indeed large and that many technologically important reacting flows occur in the flamelet regime.

In the flamelet regime, it is convenient to describe the flame-flow interactions in terms of the following two basic ingredients: a flame speed that characterizes the flame inner structure and the flame front surface area. For instance, the mean reaction rate may be written as a mean integrated chemical rate times the flame surface density:

$$
\left\langle\dot{\omega}_{R}\right\rangle=\left\langle\int \dot{\omega}_{R} d n\right\rangle_{S}\left\langle\Sigma^{\prime}\right\rangle
$$

where $\dot{\omega}_{R}$ is the mass of fuel consumed per unit time and per unit volume; $\int \dot{\omega}_{R} d n$ is the local integral of the reaction rate along the flame normal direction and characterizes the local combustion intensity; $\Sigma^{\prime}$ is the flame surface area per unit volume. The flame surface density is defined as the expected value for $\Sigma^{\prime}: \Sigma=\left\langle\Sigma^{\prime}\right\rangle$. Note that in Eq.(1), while \langle\rangle denotes a standard, unweighted ensemble-average, \langle\rangle$_{s}$ denotes a surface mean, defined as an area-weighted ensemble-average (see Section 1.2).

In Eq.(1), the integral of the reaction rate is simply related to the local fuel consumption speed, $S_{C}$, defined as:

$$
S_{C} \equiv \frac{1}{\rho_{u} Y_{R, u}} \int \dot{\omega}_{R} d n
$$

where $\rho_{u}$ and $Y_{R, u}$ are respectively the density and the fuel mass fraction in the unburnt gas. The mean reaction rate may then be expressed as the surface mean of $S_{C}$, called the flamelet speed, times the flame surface density:

$$
\left\langle\dot{\omega}_{R}\right\rangle=\left(\rho_{u} Y_{R, u}\left\langle S_{C}\right\rangle_{S}\right) \Sigma .
$$

Eq.(3) is the classical flamelet expression for the ensemble mean of the reaction rate. In general, this mean reaction rate is a function of both position and time, $\left\langle\dot{\omega}_{R}\right\rangle(\mathbf{x}, t)$. An overall reaction rate can also be obtained by space-averaging Eq.(3) over an arbitrary volume, $V$, that encloses the reaction zone:

$$
\overline{\left\langle\dot{\omega}_{R}\right\rangle}(t) \equiv \frac{1}{V} \int\left\langle\dot{\omega}_{R}\right\rangle(\mathbf{x}, t) d V=\left(\rho_{u} Y_{R, u} \bar{S}_{C}(t)\right) \frac{S_{V}(t)}{V}
$$

where $S_{V}(t)$ is the total flame surface area within the volume $V, S_{V}(t)=\int \Sigma d V$; and $\bar{S}_{C}(t)$ is a mean fuel consumption speed, $\bar{S}_{C}(t)=\int\left\langle S_{C}\right\rangle_{S} \Sigma d V / \int \Sigma d V . \bar{S}_{C}$ can also be expressed as the area-weighted, space-averaged value of $S_{C}$ integrated along the turbulent flame surface: $\bar{S}_{C}=\int S_{C} \delta A / \int \delta A$, where $\delta A$ designates the area of an infinitesimal flame element and where integration takes place along the flame surface. $\bar{S}_{C}$ and $S_{V}$ are global flame properties and are simply related to the turbulent flame speed (see Section 2.2). 
The flamelet speed, $\left\langle S_{C}\right\rangle_{S}$, in Eq.(3) and the mean fuel consumption speed, $\bar{S}_{C}$, in Eq.(4) account for local variations of the reaction rate along the flame surface. Laminar flame theory indicates that the local flame structure is modified by flow divergence, usually characterized by the strain rate acting in the flame tangent plane as well as by flame front curvature (Clavin 1985, Williams 1985). Under certain conditions, these variations can become critical and lead to partial or total quenching of the flame. Recent studies using DNS, however, suggest that quenching is a rather unlikely event for turbulent premixed flames (Poinsot et al. 1991). In addition, although the local combustion intensity may exhibit large fluctuations along the turbulent flame front, particularly for non-unity Lewis number flames, and although these fluctuations may in turn result in strong spatial variations of the flamelet speed, $\left\langle S_{C}\right\rangle_{S}$, across the turbulent flame brush, DNS suggest that these departures from the laminar consumption rate always tend to cancel in the mean (Haworth \& Poinsot 1992, Rutland \& Trouvé 1991, Trouvé 1991). In the simulations, the mean fuel consumption speed, $\bar{S}_{C}$, remain within $10 \%$ to $30 \%$ from the one-dimensional, laminar flame speed value, $s_{L}$.

Thus, it appears that in the absence of quenching, the mean fuel consumption speed, $\bar{S}_{C}$, is only weakly sensitive to the flow field, and the principle effect of turbulence is for the fluctuating velocity field to wrinkle the flame and greatly increase its surface area. This phenomenon accounts for most of the increase in the overall burning rate due to the turbulence and is represented in Eq.(3) through the flame surface density, $\Sigma$.

\subsection{The evolution equation for the flame surface density, $\Sigma$}

In current flamelet models, the flame surface density, $\Sigma$, is obtained via a modeled transport equation. This equation was first postulated by Marble \& Broadwell (1977) based on phenomenological grounds. A more rigorous approach was later proposed by Pope (1988) and Candel \& Poinsot (1990) who derive an exact balance equation for the flame surface-to-volume ratio, $\Sigma^{\prime}$ :

$$
\frac{\partial \Sigma^{\prime}}{\partial t}+\nabla \cdot \dot{\mathbf{X}} \Sigma^{\prime}=(\nabla \cdot \dot{\mathbf{X}}-\mathbf{n n}: \nabla \dot{\mathbf{X}}) \Sigma^{\prime}
$$

where $\dot{X}$ is the displacement speed of the flame surface, given by the sum of the fluid velocity and the flame propagation speed in the normal direction: $\dot{\mathbf{X}}=\mathbf{u}+w \mathbf{n} ; \mathbf{n}$ is the unit vector normal to the flame surface; and where we use tensorial notations: $(\mathbf{n n}: \nabla \dot{\mathbf{X}})=n_{i} n_{j} \frac{\partial \dot{X}_{i}}{\partial x_{j}}$.

The right-hand side of Eq.(5) can also be expressed in terms of flame stretch. The flame stretch, $k$, is defined as the rate of change of a Lagrangian flame surface element, $\delta A$ :

$$
k=\frac{d(\delta A)}{d t}=\frac{\partial(\delta A)}{\partial t}+\dot{\mathbf{X}} \cdot \nabla(\delta A)
$$

A more useful expression for $k$ is in terms of strain rate, flame curvature, and flame propagation speed (see for example Candel \& Poinsct 1990):

$$
k=a_{T}+2 w k_{m}
$$


where $a_{T}$ is the rate of strain acting in the flame tangent plane: $a_{T}=\nabla \cdot \mathbf{u}-\mathbf{n n}: \nabla \mathbf{u}$; and $k_{m}$ is the flame surface curvature, as given by the divergence of the flame normal direction: $2 k_{m}=\nabla$.n. In Eq.(7), positive curvature is chosen convex towards the reactants.

Using Eq.(7), the balance equation for the flame surface-to-volume ratio can be re-written as:

$$
\frac{\partial \Sigma^{\prime}}{\partial t}+\nabla \cdot \dot{\mathbf{X}} \Sigma^{\prime}=k \Sigma^{\prime}
$$

When ensemble-averaged, this equation yields an exact balance equation for the flame surface density (Pope 1988, Cant et al. 1990):

$$
\frac{\partial \Sigma}{\partial t}+\nabla \cdot\langle\dot{\mathbf{X}}\rangle_{S} \Sigma=\langle k\rangle_{S} \Sigma
$$

where the flame surface mean of any quantity $Q$ is given by: $\langle Q\rangle_{S}=\left\langle Q \Sigma^{\prime}\right\rangle /\left\langle\Sigma^{\prime}\right\rangle=$ $\left\langle Q \Sigma^{\prime}\right\rangle / \Sigma$. Note that surface means are different from standard means. In particular, the surface mean of a quantity $Q$ is different from the ensemble mean of $Q$ conditioned on being at the flame location (see Section 2.1).

Eq.(9) can be cast in various forms. For modeling purposes, it is useful to split the velocity vector into a mean component and a turbulent fluctuation: $\mathbf{u}=\langle\mathbf{U}\rangle+\mathbf{u}^{\prime}$. We can then re-write Eq.(9) as follows:

$$
\frac{\partial \Sigma}{\partial t}+\nabla \cdot\langle\mathbf{U}\rangle \Sigma+\nabla \cdot\left\langle\mathbf{u}^{\prime}\right\rangle_{S} \Sigma+\nabla \cdot\langle w \mathbf{n}\rangle_{S} \Sigma=\left\langle a_{T}\right\rangle_{S} \Sigma+\left\langle A_{T}\right\rangle_{S} \Sigma+2\left\langle w k_{m}\right\rangle_{S} \Sigma,
$$

where we use the following notations:

$$
\begin{gathered}
\left\langle a_{T}\right\rangle_{S}=\left\langle\nabla \cdot \mathbf{u}^{\prime}-\mathbf{n n}: \nabla \mathbf{u}^{\prime}\right\rangle_{S}, \\
\left\langle A_{T}\right\rangle_{S}=\nabla\langle\mathbf{U}\rangle-\langle\mathbf{n n}\rangle_{S}: \nabla\langle\mathbf{U}\rangle .
\end{gathered}
$$

The three convective terms on the left-hand side of Eq.(10) are transport terms that correspond respectively to convection by the mean flow, turbulent diffusion, and flame propagation. The terms on the right-hand side of the equation are the source and sink terms for the flame surface density: $\left\langle a_{T}\right\rangle_{S}$ is the turbulent strain rate acting in the flame tangent plane, $\left\langle A_{T}\right\rangle_{S}$ is the strain rate due to the mean flow field, and $2\left\langle w k_{m}\right\rangle_{S}$ is a term that accounts for the combined effects of flame curvature and flame propagation.

Eq.(10) is an exact expression of the evolution equation for the flame surface density, $\Sigma$, in a turbulent flow field. Unfortunately, many terms remain unclosed in this exact turbulent $\Sigma$-equation. Modeling assumptions are, therefore, required, in particular to calculate the turbulent diffusion term and the turbulent flame stretch. Different formulations of the modeled $\Sigma$-equation have been proposed in the literature (Marble \& Broadwell 1977, Darabiha et al. 1987, Maistret et al. 1989, Candel et al. 1990, Cant et al. 1990, Borghi 1990, Mantel \& Borghi 1991). However, since the turbulent flame stretch as well as the flame surface density is not available 
from experiments, little is known on the validity of the closure assumptions used in current flamelet models. DNS is an attractive alternate approach to get basic information on these fundamental quantities. In the present work, three-dimensional DNS of premixed flames in isotropic turbulent flow is used to estimate the different terms appearing in the $\Sigma$-equation, with particular emphasis on the source and sink terms on its right-hand side that determines production or dissipation of flame surface area.

\subsection{Direct Numerical Simulation of turbulent premixed flames}

We use DNS to analyze the different terms in the equation for the flame surface density. The simulations are performed using a three-dimensional, compressible Navier-Stokes solver that fully resolves the turbulent flow field. Spatial derivatives are computed with a modified Padé scheme that is sixth-order accurate (Lele 1992). Solutions are advanced in time using a third-order Runge-Kutta method (Wray 1990). Boundary conditions are specified using the NSCBC method (Poinsot \& Lele 1992). Because of the otherwise prohibitive computational cost, simulations are limited to simple but finite-rate reaction schemes. In this work, the chemistry model is a single step, irreversible chemical reaction where the reaction rate depends exponentially on temperature (Arrhenius kinetics):

$$
\dot{\omega}_{R}=B \rho Y_{R} \exp \left(-\frac{T_{a}}{T}\right)
$$

where $T_{a}$ is the activation temperature and $B$ a constant that depends on the selected flame speed. This formulation corresponds to a binary reaction in which one of the reactants, $Y_{R}$, is strongly deficient as, for example, in fuel-lean combustion. Also, it is worth emphasizing that the simulations are not limited by the constant density assumption, and heat release effects are fully accounted for.

Following Williams (1985), we re-write the reaction rate as:

$$
\dot{\omega}_{R}=\Lambda \rho Y_{R} \exp \left(\frac{-\beta(1-\Theta)}{1-\alpha(1-\Theta)}\right)
$$

where $\Theta$ is the reduced temperature, $\Theta=\left(T-T_{u}\right) /\left(T_{b}-T_{u}\right) ; T_{u}$ is the temperature of the fresh reactants; $T_{b}$ is the adiabatic flame temperature; and the coefficients $\Lambda, \alpha$, and $\beta$ are, respectively, the reduced pre-exponential factor, the heat release factor, and the reduced activation energy:

$$
\Lambda=B \exp (-\beta / \alpha), \quad \alpha=\left(T_{b}-T_{u}\right) / T_{b}, \quad \text { and } \quad \beta=\alpha T_{a} / T_{b}
$$

In the following, we use $\alpha=0.75$ and $\beta=8$.

Another important feature of the simulations is that the transport coefficients are temperature dependent. These coefficients satisfy the following relations:

$$
\mu=\mu_{u}\left(T / T_{u}\right)^{b}, L e=\lambda / \rho D c_{p}=\text { constant, } \operatorname{Pr}=\mu c_{p} / \lambda=\text { constant }
$$


where $\mu, \lambda$, and $D$ are the molecular diffusivities of, respectively, momentum, internal energy, and species mass, $b$ is a constant, and $L e$ and $\operatorname{Pr}$ are, respectively, the Lewis number and the Prandtl number. We use $b=0.76, \operatorname{Pr}=0.75$. Simulations have been performed for different Lewis numbers, $L e=0.8,1.0$, and 1.2.

The selected computational configuration corresponds to a premixed flame embedded in a three-dimensional, decaying, isotropic turbulent flow. The left- and right-hand sides of the computational domain are inflow and outflow boundaries, while periodic boundary conditions are applied at lateral walls. The calculations are initialized with fresh reactants on one side of the domain and burnt products on the other side; the two are separated by a plane laminar flame. Isotropic turbulence is initially located in the flow of fresh reactants, its velocity field being specified according to a model spectrum. The turbulence is characterized by a Kolmogorov length scale smaller than the thermal thickness of the laminar flame, $\eta_{k} / \delta_{T}=0.1$ where $\delta_{T}=\left(T_{b}-T_{u}\right) /(d T / d x)_{\max }$, and a turbulence intensity that is much higher than the laminar flame speed, $u^{\prime} / s_{L}=10$. The initial turbulent Reynolds number, based on the Taylor microscale, is 50 . The initial turbulent Reynolds number, based on the integral length scale, is 70 . The grid resolution is $129^{3}$.

The simulations describe the wrinkling of the flame zone due to turbulent motions as well as the combustion feedback due to dilatation and temperature-dependent transport properties. Note that the turbulence is decaying in time, and flow conditions are non-stationary.

\section{Accomplishments}

\subsection{Diagnostics}

All terms appearing in Eq.(10) can be obtained from the simulations. We now briefly describe how. The velocity vector and the velocity gradient tensor are readily obtained from the resolved flow field. To define flame-based quantities, we make use of concepts based on the thin flame picture. First, a progress variable, $c$, is introduced to indicate location within the reaction zone, $c=1-Y_{R}$, where $Y_{R}$ is the normalized fuel mass fraction. The progress variable varies monotonically through the flame from 0 in the reactants to 1 in the products. Constant progress variable surfaces may conveniently be used to define the flame front location: we use the surface $c=c_{f}=0.8$. In addition, at any location on this surface, the local gradient of $c$ defines the normal direction to the flame front:

$$
\mathbf{n}=-\frac{\nabla c}{|\nabla c|}
$$

where $\mathbf{n}$ points into the fresh reactants.

The propagation speed of the flame surface, $w$, is obtained from an expression analog to the well-known field equation (also called the $G$-equation, see for example Kerstein et al. 1988). Let us first consider a point on the flame surface, $c=c_{f}$. The velocity, $\dot{\mathbf{X}}$, at which this point must move to remain on the surface, is given by:

$$
\frac{\partial c}{\partial t}+\dot{\mathbf{X}} \cdot \nabla c=0
$$


which, using Eq.(15), implies that:

$$
\dot{\mathbf{X}} \cdot \mathbf{n}=\frac{1}{|\nabla c|} \frac{\partial c}{\partial t}
$$

and yields the following expression for the flame propagation speed:

$$
w=\dot{\mathbf{X}} \cdot \mathbf{n}-\mathbf{u} \cdot \mathbf{n}=\frac{1}{|\nabla c|} \frac{D c}{D t},
$$

where the quantities are estimated at the surface $c=c_{f}$. In the simulations, we solve for a conservation equation for the fuel mass fraction, $Y_{R}$, and since by definition $c=1-Y_{R}$, the expression above can be readily obtained from the DNS data base.

The flame surface density, $\Sigma$, is a more subtle quantity. It includes both geometrical and statistical information. Following Pope (1990), the flame surface density is expressed as the product of the expected value for the magnitude of the gradient of $c$, conditioned on being on the flame surface, times the probability of being on that surface:

$$
\Sigma^{\prime}=|\nabla c| \delta\left(c-c_{f}\right), \quad \text { and } \quad \Sigma=\left\langle\Sigma^{\prime}\right\rangle=\left\langle|\nabla c| \mid c=c_{f}\right\rangle p\left(c_{f}\right)
$$

where $p\left(c_{f}\right)$ is the probability of $c=c_{f}$. An equivalent expression for $\Sigma$, also proposed by Pope (1990), is in terms of the joint probability of the scalar $c$ and the magnitude of its gradient:

$$
\Sigma=\int_{0}^{\infty} \gamma p\left(c_{f}, \gamma\right) d \gamma
$$

where $\gamma=|\nabla c|$.

We now turn to the averaging problem. In the simulations, the flame brush propagates along the $x$ direction, and the problem remains homogeneous in the $y-z$ planes. Therefore, averaged quantities depend on $x$ and time $t$ only, and ensemble-averaging can be performed in the $y-z$ planes:

$$
\langle Q\rangle(x, t)=\frac{1}{L_{y} L_{z}} \int Q(x, y, z, t) d y d z,
$$

where $L_{y}$ and $L_{z}$ are the $y$ and $z$ dimensions of the computational domain. Since the flow field is non-stationary, time-averaging cannot be used and the present analysis is based exclusively on the slicing of the DNS data base in planes perpendicular to the direction of mean flame propagation.

Let us now consider a particular $y-z$ plane, $x=x_{0}$. In that plane, the flame contour $c=c_{f}$ is a set of closed lines. The flame surface density as well as the various surface and conditional means in the analysis can be expressed in terms of line-integrals along this $\left(c=c_{f}, x=x_{0}\right)$ line-contour. For instance, as suggested by Eq. $(20)$, the flame surface density, $\Sigma$, can be obtained via the joint probability 


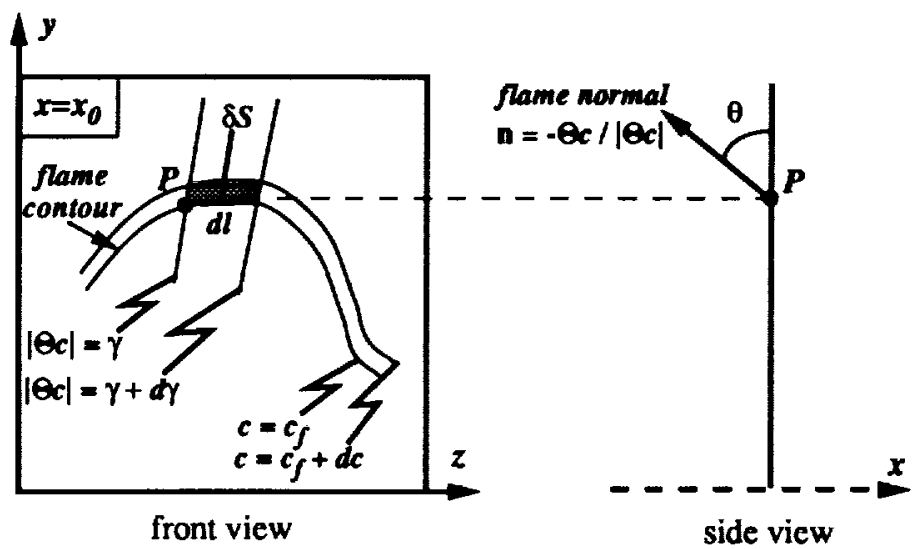

Figure 1. A geometrical expression for the joint probability of the progress variable and the magnitude of its gradient in a given $y-z$ plane: $p\left(c_{f}, \gamma\right) d c d \gamma=$ $\operatorname{prob}\left(c_{f} \leq c \leq c_{f}+d c ; \gamma \leq|\nabla c| \leq \gamma+d \gamma\right)=\delta S / L_{y} L_{z}$, see Eq.(22).

$p\left(c_{f}, \gamma\right)$. Simple geometrical considerations lead to the following relation (Figure $1)$ :

$$
p\left(c_{f}, \gamma\right) d \gamma=\frac{1}{L_{y} L_{z}} \sum_{j=1}^{J} \frac{d l}{\gamma|\cos \theta|}
$$

where $d l$ designates the arc-length of an infinitesimal flame element on the linecontour $c=c_{f}$ and $\theta$ is a measure of the orientation of this flame element with respect to the $x=x_{0}$ plane. In Eq.(22), summation is over all occurrences of the event $(c,|\nabla c|)=\left(c_{f}, \gamma\right)$. Eqs.(20) and (22) then give the following expression for $\Sigma$ :

$$
\Sigma=\frac{1}{L_{y} L_{z}} \int_{c=c_{f}} \frac{d l}{|\cos \theta|}=\frac{1}{L_{y} L_{z}} \int_{c=c_{j}} \frac{|\nabla c|}{\sqrt{\left(\frac{\partial c}{\partial y}\right)^{2}+\left(\frac{\partial c}{\partial z}\right)^{2}}} d l,
$$

where we use the geometrical relation: $|\cos \theta|=\sqrt{\left(\frac{\partial c}{\partial y}\right)^{2}+\left(\frac{\partial c}{\partial z}\right)^{2}} /|\nabla c|$.

Eq.(23) differs from the original formulation proposed by Trouvé \& Poinsot (1992) due to an error in their original expressions for surface means and conditional means. However, their estimate of the probability $p\left(c_{f}\right)$ remains unchanged:

$$
p\left(c_{f}\right)=\int_{0}^{\infty} p\left(c_{f}, \gamma\right) d \gamma=\frac{1}{L_{y} L_{z}} \int_{c=c_{f}} \frac{d l}{|\cos \theta||\nabla c|}=\frac{1}{L_{y} L_{z}} \int_{c=c_{j}} \frac{d l}{\sqrt{\left(\frac{\partial c}{\partial y}\right)^{2}+\left(\frac{\partial c}{\partial z}\right)^{2}}}
$$

and, compared to the new formulation, the results of Trouve \& Poinsot (1992) still hold within a few percents.

Using Eq.(22), the correct expression for conditional means is as follows:

$$
\left\langle Q \mid c=c_{f}\right\rangle(x, t)=\frac{\int_{c=c_{\rho}} Q(|\cos \theta||\nabla c|)^{-1} d l}{\int_{c=c_{\rho}}(|\cos \theta||\nabla c|)^{-1} d l} .
$$


The surface means are then obtained using the following relations:

$$
\langle Q\rangle_{S}(x, t)=\frac{\left\langle Q \Sigma^{\prime}\right\rangle}{\left\langle\Sigma^{\prime}\right\rangle}=\frac{\left\langle Q|\nabla c| \mid c=c_{f}\right\rangle}{\left\langle|\nabla c| \mid c=c_{f}\right\rangle}=\frac{\int_{c=c_{f}} Q|\cos \theta|^{-1} d l}{\int_{c=c_{f}}|\cos \theta|^{-1} d l}
$$

Clearly, surface means differ from conditional means.

The relations above provide a methodology to estimate the different means needed in our analysis. The accuracy of these expressions depends on the size of the computational domain with respect to the turbulent length scales. In the simulations, the integral length scale of the turbulent flow field grows as the kinetic energy decays; this growth, however, is rather slow, and it has been determined that the integral length scale remains at least 8 times smaller than $L_{y}$ and $L_{z}$. Although we recognize that the statistics are somewhat undersampled, reasonable accuracy is expected when estimating the first moments.

\subsection{Global flame properties}

As described in the previous section, the analysis takes advantage of the fact that the problem is statistically one-dimensional and provides the various quantities of interest as a function of time $t$ and position $x$ within the turbulent flame brush. We now discuss briefly the relationship between these spatially-resolved quantities and the more familiar, global (space-averaged) flame properties.

By definition, the total flame surface area, $S_{V}$, within the computational domain is equal to the volume-integral of the flame surface density, which, in the present case, translates to the following relation:

$$
\frac{S_{V}(t)}{L_{y} L_{z}}=\int \Sigma(x, t) d x
$$

Using Eq.(9), it can be shown that the time-derivative of the relative increase in flame surface area is equal to the total flame stretch, $\bar{k}$, space-averaged across the turbulent flame brush:

$$
\bar{k}(t)=\frac{d}{d t}\left(\frac{S_{V}(t)}{L_{y} L_{z}}\right)=\int\langle k\rangle_{S}(x, t) \Sigma(x, t) d x .
$$

$\bar{k}$ gives the instantaneous rate of change of the total flame surface area in the computational domain. If positive, the flame surface grows; if negative, the flame surface contracts.

The mean fuel consumption speed, $\bar{S}_{C}$, can also be expressed in terms of spaceintegrals of the flamelet speed, $\left\langle S_{C}\right\rangle_{S}$, and the flame surface density, $\Sigma$, across the turbulent flame brush:

$$
\bar{S}_{C}(t)=\frac{\int\left\langle S_{C}\right\rangle_{S}(x, t) \Sigma(x, t) d x}{\int \Sigma(x, t) d x}
$$


Finally, using Eq.(4), the turbulent flame speed, $S_{T}$, is simply related to the product of $S_{V}$ and $\bar{S}_{C}$ :

$$
S_{T}(t)=\frac{1}{\rho_{u} Y_{R, u}} \int\left\langle\dot{\omega}_{R}\right\rangle(x, t) d x=\int\left\langle S_{C}\right\rangle_{S}(x, t) \Sigma(x, t) d x=\bar{S}_{C}(t) \frac{S_{V}(t)}{L_{y} L_{z}} .
$$

As described in the previous sections, the present study uses three-dimensional direct numerical simulations of turbulent premixed flames in isotropic flow. Three different cases have been studied that correspond to turbulent flames characterized by the same laminar thermal thickness, $\delta_{T}$, the same laminar flame speed, $s_{L}$, embedded in the same initial turbulent flow field, but with different Lewis number, $L e=0.8,1.0$, and 1.2 .

The three cases exhibit large differences in the time history of the turbulent flame speed, $S_{T}(t)$. As suggested by Eq.(30), these differences may be related to a modification of the turbulent flame structure through changes in $\bar{S}_{C}$, to a modification of the total flame surface area through changes in $S_{V}$, or to a combination of both. In fact, irrespective of the value of the Lewis number and in agreement with the findings from previous studies (Ashurst et al. 1987, Haworth \& Poinsot 1992, Rutland \& Trouvé 1991), the mean fuel consumption speed, $\bar{S}_{C}$, is found to be only a weak function of the turbulence. At all times, departures of $\bar{S}_{C}$ from the laminar value, $s_{L}$, remain within $20 \%$. This result implies that the large effect of the Lewis number on $S_{T}$ observed in the simulations is primarily a flame surface effect and that the flame surface density is the key quantity that to first order determines the mean reaction rate. For more details, see Trouvé \& Poinsot (1992).

\subsection{Comparison of DNS results with flamelet models}

Using the methodology described in Section 2.1 , we now analyze the spatial distribution of flame surface density across the turbulent flame brush. Figure 2 compares several $\Sigma$-profiles taken at different instants in the simulations. The Lewis number is 0.8 . At $t=0, \Sigma$ is a delta function located at $x=0$. As time evolves, the turbulent flame brush gets thicker and propagates deeper into the reactants. Accordingly, the $\Sigma$-profile spreads out and shifts towards negative values of $x$. In the simulations, this shift is rather weak but can clearly be seen at the latest times $(t=6 \tau$ in Figure 2).

Figure 3 compares the $\Sigma$-profiles obtained for different Lewis numbers. The comparison is performed at the same instant in the simulations, $t=4 \tau$. The effect of the Lewis number is clearly seen in this plot with a turbulent flame brush that propagates faster and deeper into the reactants for more diffusive mixtures. Note that as shown in Eq.(27), the $x$-integral of $\Sigma$ gives the relative increase of total flame surface area and the local differences in the profiles plotted in Figure 3, when integrated, correspond to large global differences in the total amount of flame surface area, $S_{V}$.

A different perspective is adopted in Figure 4 where $x$-location within the turbulent flame brush is indicated by the local mean progress variable, $\langle c\rangle$. Although they are different in physical space, the $\Sigma$-profiles obtained for different Lewis numbers collapse when mapped in $\langle c\rangle$-space. This result indicates that the effect of the 


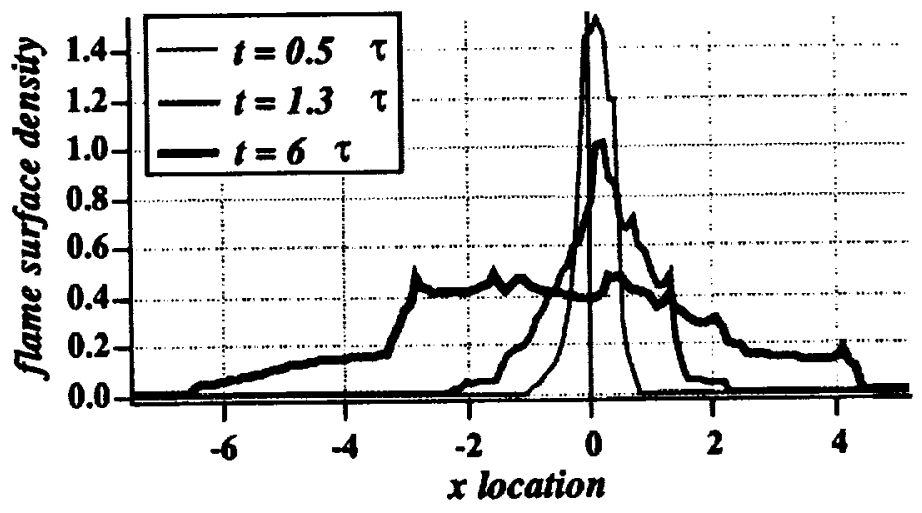

FIGURE 2. Time evolution of the flame surface density, $\Sigma$, in the computational domain (reactants on the left; products on the right). $L e=0.8$. $\Sigma$ and $x$ are made non-dimensional by the laminar thermal thickness, $\delta_{T}$. Time is measured in units of the initial turbulent eddy turn-over time, $\tau$.

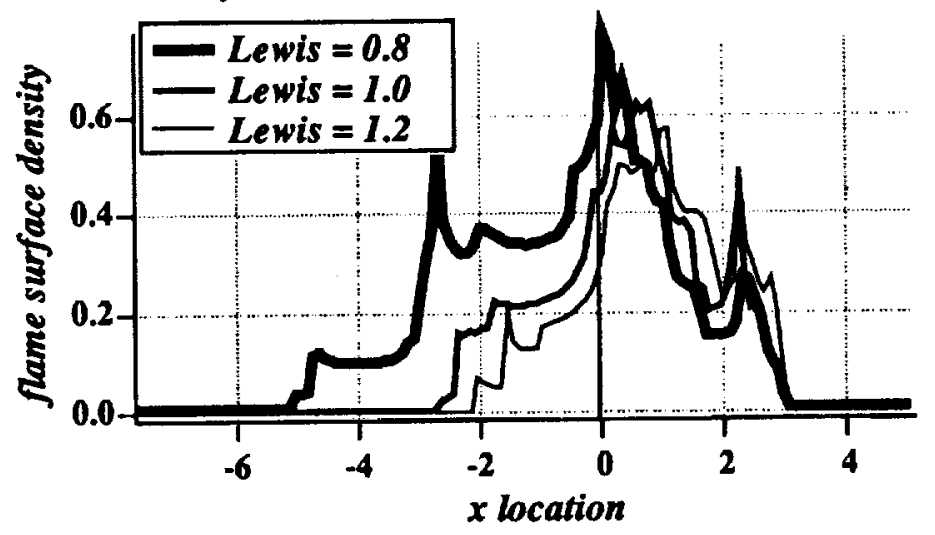

FIGURE 3. Lewis number effects on the spatial distribution of flame surface density, $\Sigma$, in the computational domain (reactants on the left; products on the right). $t=4 \tau, \Sigma$ and $x$ are made non-dimensional by the laminar thermal thickness, $\delta_{T}$.

Lewis number on the spatial distribution of flame surface density is primarily an effect on the turbulent flame thickness.

Also plotted in Figure 4 is the flame surface density predicted by the BrayMoss-Libby (BML) model. The BML model is a well-documented, second order closure model designed to avoid gradient transport assumptions (see for example Bray 1980, and 1990, Libby \& Bray 1980). The model pre-assumes a bimodal shape for the probability density function of the progress variable, an assumption that is consistent with a laminar flamelet description of the reaction zone and represents the intermittent presence of packets of unburnt and fully burnt mixture at any location within the turbulent flame brush. Various degrees of refinement are possible within 


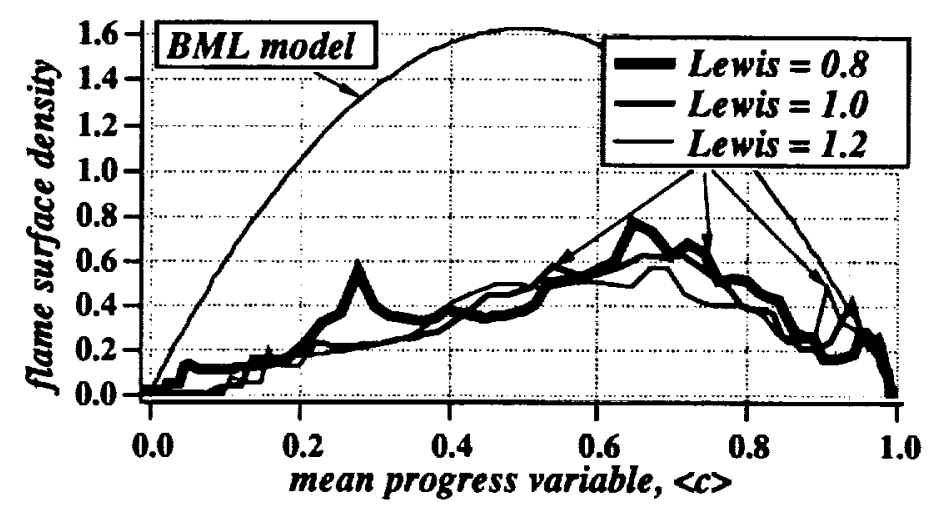

FIGURE 4. The spatial distribution of flame surface density, $\Sigma$, across the turbulent flame brush: comparison between DNS and the Bray-Moss-Libby model. $t=4 \tau . \Sigma$ is made non-dimensional by the laminar thermal thickness, $\delta_{T}$.

the BML framework, in particular with respect to the chemical source terms and the flame surface density. Although a transport equation for $\Sigma$ could be incorporated easily (Cant et al. 1990), current formulations of the model are limited to a simple algebraic closure (Bray et al. 1984, 1989). The expression for $\Sigma$ in a $y-z$ plane, $x=x_{0}$, where $\langle c\rangle=$ constant, is:

$$
\Sigma=\frac{g\langle c\rangle(1-\langle c\rangle)}{\bar{\sigma}_{y} \hat{L}_{y}}
$$

where $g$ is a constant of order unity, $\hat{L}_{y}$ is a length that characterizes the flame wrinkling, and $\bar{\sigma}_{y}$ is the mean of a direction cosine defining the flamelet orientation relative to the plane, $x=x_{0}$. Both $\hat{L}_{y}$ and $\bar{\sigma}_{y}$ are assumed to be constant across the turbulent flame brush. $\hat{L}_{y}$ is specified empirically as follows:

$$
\hat{L}_{y}=C_{L} l\left(\frac{s_{L}}{u^{\prime}}\right)^{n},
$$

where $l$ is the integral length scale of the turbulence, and $C_{L}$ and $n$ are model constants. Following Bray (1990), we use $n=1, C_{L}=1, g=1.5, \bar{\sigma}_{y}=0.5$.

Figure 4 shows that the structure of the $\Sigma$-profile is not well captured by the BML model which predicts a symmetric distribution of $\Sigma$ in $\langle c\rangle$-space, whereas DNS gives a skewed profile with a maximum close to $\langle c\rangle=0.7$. Furthermore, although the comparison between DNS and the BML model is rendered difficult by the turbulence decay, some qualitative observations can still be made about the accuracy or inaccuracy of the model. For instance, the BML predictions always overestimate the flame surface densities across the turbulent flame brush by a factor decreasing from more than 5 to approximately 2 in the course of the simulations. Although the exact magnitude of the error might not be meaningful, the fact that it is timedependent underscores the limits of an algebraic closure that cannot account for memory effects and is, therefore, expected to perform poorly in transient problems. 


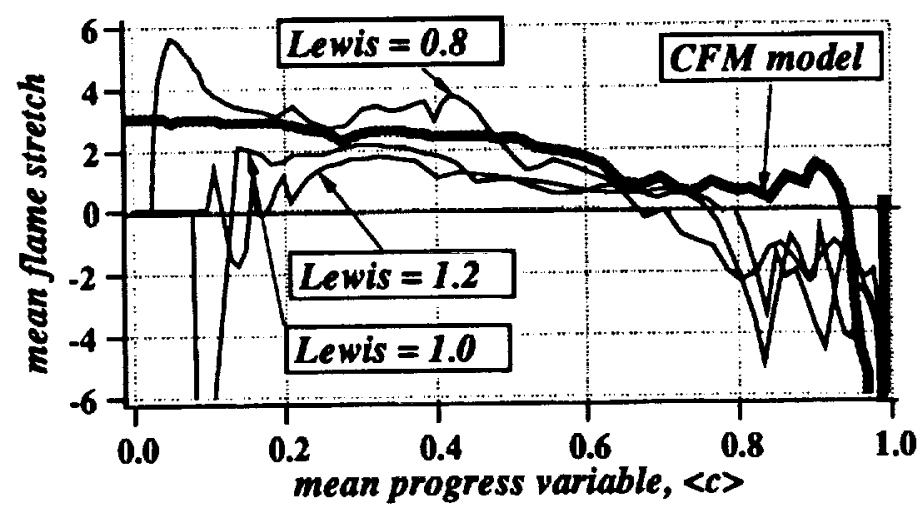

FIgURE 5. The spatial distribution of turbulent flame stretch, $\langle k\rangle_{s}$, across the turbulent flame brush: comparison between DNS and the Coherent Flame Model. $t=4 \tau . k$ is made non-dimensional by the laminar flame time, $\delta_{T} / s_{L}$.

We now turn to the more promising approach based on a modeled turbulent $\Sigma$-equation. Different formulations of this equation have been proposed in the literature (Marble \& Broadwell 1977, Darabiha et al. 1987, Maistret et al. 1989, Candel et al. 1990, Cant et al. 1990, Borghi 1990, Mantel \& Borghi 1991). These models are based on the exact but unclosed formulation given in Eq.(10). The right-hand side of Eq.(10) is the total flame stretch, which can be decomposed into a strain term and a propagation term. While the strain term, $\left\langle a_{T}\right\rangle_{S}$, remains approximately constant through the turbulent flame, the propagation term, $2\left\langle w k_{m}\right\rangle_{s}$, exhibits strong variations and decreases from positive values on the unburnt side, close to $\langle c\rangle=0$, to negative values on the burnt side, close to $\langle c\rangle=1$ (Trouvé \& Poinsot 1992). Interestingly enough, both contributions to stretch have the same order of magnitude.

Figure 5 presents the mean flame stretch profile, $\langle k\rangle_{S}$, across the turbulent reaction zone, plotted for different Lewis numbers at $t=4 \tau$. At the leading edge, strain rate and flame propagation effects are locally cumulative, and the overall balance is strongly positive. The leading edge of the turbulent flame is a region of strong production of flame surface area. On the contrary, the propagation term takes large negative values on the burnt side. In that region, strain rate and flame propagation effects are locally opposite, and the overall balance is negative. The rear edge of the turbulent flame thus appears as a region where flame surface area gets strongly dissipated. In addition, while the effect of the Lewis number is not visible on the burnt side of the turbulent flame, there are large differences between the cases $L e=0.8,1.0$, and 1.2 close to $\langle c\rangle=0$. This suggests that the turbulent flame is most sensitive to Lewis number effects on the unburnt side, whereas it remains relatively unaffected on the burnt side.

Using Eq. (28), one can quantify the overall differences due to the Lewis number; we find (in units of the laminar flame time): $\bar{k}=2.6$ for $L e=0.8$, and the flame surface area is quickly growing; $\bar{k} \simeq 0.0$ for $L e=1.0$ and $L e=1.2$, and the flame 
surface area remains roughly constant.

Also plotted in Figure 5 is a comparison with the Coherent Flame Model proposed originally by Marble \& Broadwell (1977) and further developed by Candel and his co-workers (Darabiha et al. 1987, Maistret et al. 1989, Candel et al. 1990). In this formulation, the turbulent flame stretch is written as:

$$
\langle k\rangle_{S}=\frac{\epsilon}{k_{t}}-s_{L} \frac{\Sigma}{\left\langle Y_{R}\right\rangle}
$$

where $k_{t}$ is the turbulent kinetic energy and $\epsilon$ its dissipation, both measured in the unburnt gas; $\left\langle Y_{R}\right\rangle$ is the ensemble-averaged fuel mass fraction. The first term on the right-hand side of Eq.(33) represents the straining due to the flow motions and is assumed to scale with the integral time scale of the turbulence; the second term is a disparition term associated with flame propagation and is assumed to scale with the laminar flame speed, $s_{L}$, and the flame surface density, $\Sigma$.

Figure 5 shows that this model is indeed able to reproduce qualitatively the spatial structure of the balance between production and dissipation of $\Sigma$, going from production at the leading edge of the turbulent flame to dissipation at the rear edge. However, the $1 /\left\langle Y_{R}\right\rangle$ behavior of the disparition term leads to numerical difficulties on the burnt side of the flame. The model, therefore, overpredicts the dissipation of $\Sigma$ near $\langle c\rangle=1$ and gives a negative mean flame stretch, $\bar{k}=-3.6$, in strong disagreement with the values reported above. In addition, the disparition term in Eq.(33) is always and everywhere negative and cannot account for the possible transition to unstable thermo-diffusive conditions as observed in the simulations (Trouvé \& Poinsot 1992).

\section{Conclusion and future plans}

In flamelet models, the flame surface density is a central ingredient that conveys most of the effects of the turbulence on the rate of energy release. The flame surface density can be obtained using an algebraic closure, as in current formulations of the Bray-Moss-Libby model, or via a modeled transport equation, called the $\Sigma$-equation. The $\Sigma$-equation was first postulated by Marble \& Broadwell (1977) based on phenomenological grounds. Recent theoretical work has produced a more rigorous approach that leads to an exact but unclosed formulation for the turbulent $\Sigma$-equation (Pope 1988, Candel \& Poinsot 1990). Since the flame surface density and the different terms of interest in the turbulent $\Sigma$-equation are not experimentally accessible, little is known on the validity of the closure assumptions used in current formulations of flamelet models.

Direct Numerical Simulation (DNS) is an attractive alternative approach to get basic information on these fundamental quantities. In the present work, threedimensional DNS of premixed flames propagating in isotropic turbulent flow have been used to estimate the different terms appearing in the $\Sigma$-equation. A new methodology has been proposed to provide the flame surface density, $\Sigma$, as well as the source and sink terms for $\Sigma$, estimated as a function of time and position within 
the turbulent flame brush. Using this methodology, comparisons with flamelet models are possible at a level of detail never reached before.

Principal findings are that: (1) the Bray-Moss-Libby formulation overestimates the flame surface densities; more importantly, the error is a function of time, which underscores the limits of using an algebraic closure; (2) the Coherent Flame Model (CFM), based on a modeled turbulent $\Sigma$-equation, is shown capable of reproducing qualitatively the spatial variations in the balance between production and dissipation of flame surface area - the leading edge of the turbulent flame is correctly described as a region of production of flame surface area, whereas the rear edge is a region where flame surface gets strongly dissipated - however, the CFM model tends to overpredict flame surface dissipation and is unable to reproduce variations due to thermo-diffusive phenomena.

Ongoing plans include the development of a new formulation of the $\Sigma$-equation that would incorporate thermo-diffusive mechanisms. Also, the effect of the mean flow field as represented by $\left\langle A_{T}\right\rangle_{S}$ in Eq.(10) is currently studied using threedimensional DNS of a turbulent flame embedded in homogeneous shear flow.

Finally, it is worth emphasizing that theoretical descriptions of turbulent combustion are aimed at providing tractable expressions for the following two categories of unclosed terms: (1) the mean chemical source term; (2) the double and/or triple correlations representing turbulent transport phenomena. We have focused exclusively so far on the first category of problems, i.e. predicting the mean reaction rate. The second category is also a promising field for DNS of turbulent combustion. Indeed, in most current models, turbulent transport terms are treated using conventional, first-order closure assumptions based on the concept of an eddy viscosity. However, since flames of practical interest feature large changes in mass density which affect significantly the turbulent flow field, it is somewhat suspicious, although very common, to directly extend the empirical closures developed in the context of constant density, non-reacting flow problems to the case of turbulent flames. Future work will include studying and modeling of turbulent transport in a combustion environment.

\section{Acknowledgements}

Fruitful and friendly interactions with other members of the combustion group at CTR are gratefully acknowledged. In particular, we thank Dr. Feng Gao and Dr. Luc Vervisch.

\section{REFERENCES}

Abdel-Gayed, R. G., Bradley, D., Hamid, M. N., \& LaWes, M. 1984 Lewis number effects on turbulent burning velocity. Twentieth Symp. (International) on Combust. 505-512. The Combustion Institute.

Ashurst, W. T., Peters, N., \& Smooke, M. D. 1987 Numerical simulation of turbulent flame structure with non-unity Lewis number. Combust. Sci. Technol. 53, 339-375. 
BORGHI, R. 1985 On the structure and morphology of turbulent premixed flames. in Recent Advances in Aerospace Sciences. Pergamon.

BORGHI, R. 1990 Turbulent premixed combustion: further discussion on the scales of the fluctuations. Combust. Flame. 80, 304-312.

Bray, K. N. C. 1980 Turbulent flows with premixed reactants. in Topics in Applied Physics 44. Springer-Verlag.

Bray, K. N. C., LibBy, P. A., \& Moss, J. B. 1984 Flamelet crossing frequencies and mean reaction rates in premixed turbulent combustion. Combust. Sci. Technol. 41, 143-172.

Bray, K. N. C., Libby, P. A., \& Moss, J. B. 1984 Scalar length scale variations in premixed turbulent flames. Twentieth Symp. (International) on Combust. 421-427. The Combustion Institute.

Bray, K. N. C., \& LiBby, P. A. 1986 Passage times and flamelet crossing frequencies in premixed turbulent combustion. Combust. Sci. Technol. 47, 253274.

Bray, K. N. C., Champion, M., \& Libby, P. A. 1989 The interaction between turbulence and chemistry in premixed turbulent flames. in Turbulent Reactive Flows, Lecture Notes in Eng. 40. Springer-Verlag.

Bray, K. N. C. 1990 Studies of the turbulent burning velocity. Proc. R. Soc. Lond. A. 431, 315-335.

Candel, S. M., Veynante, D., Lacas, F., Maistret, E., Darabiha, N., \& POINSOT, T. 1990 Coherent flame model: applications and recent extensions. in Series on Advances in Mathematics for Applied Sciences. World Scientific.

CANDEL, S. M., \& PoInsot, T. 1990 Flame stretch and the balance equation for the flame surface area. Combust. Sci. Technol. 70, 1-15.

Cant, R. S., Pope, S. B., \& Bray, K. N. C. 1990 Modelling of flamelet surfaceto-volume ratio in turbulent premixed combustion. Twenty-Third Symp. (International) on Combust. 809-815. The Combustion Institute.

Clavin, P. 1985 Dynamic behavior of premixed flame fronts in laminar and turbulent flows. Prog. Energy Combust. Sci. 11, 1-59.

Darabiha, N., Giovangigli, V., Trouvé, A., Candel, S. M., \& Esposito, E. 1987 Coherent flame description of turbulent premixed ducted flames. in Proc. of the France-USA Joint Workshop on Turbulent Combustion. Springer Verlag.

Haworth, D. C., \& Poinsot, T. 1992 Numerical simulations of Lewis number effects in turbulent premixed flames. J. Fluid Mech. 244, 405-436.

Kerstein, A. R., Ashurst, W. T., \& Williams, F. A. 1988 Field equation and interface propagation in an unsteady homogeneous flow field. Phys. Rev. A. 37, 2728-2731.

LELE, S. K. 1992 Compact finite difference schemes with spectral-like resolution. J. Comp. Phys. 103, 16-42. 
Libby, P. A., \& BraY, K. N. C. 1980 Implications of the laminar flamelet model in premixed turbulent combustion. Combust. Flame. 39, 33-41.

Maistret, E., Darabiha, N., Poinsot, T., Veynante, D., Lacas, F., CanDEL, S. M., \& EsPosito, E. 1989 Recent developments in the coherent flamelet description of turbulent combustion. in Proc. Srd Int. SIAM Conf. on Numerical Combustion.

Mantel, T., \& Borghi, R. 1991 A new model of premixed wrinkled flame propagation based on a scalar dissipation equation. in Proc. 19th ICDERS.

Marble, F. E., \& Broadwell, J. E. 1977 The coherent flame model for turbulent chemical reactions. Project Squid Technical Report. TRW-9-PU.

PETERS, N. 1986 Laminar flamelet concepts in turbulent combustion. Twenty-First Symp. (International) on Combust. 1231-1250. The Combustion Institute.

Poinsot, T., Veynante, D., \& CANDel, S. M. 1991 Quenching processes and premixed turbulent combustion diagrams. J. Fluid Mech. 228, 561-605.

Poinsot, T., EChekki, T., \& Mungal, M. G. 1992 A study of the laminar flame tip and implications for premixed turbulent combustion. Combust. Sci. Technol. 81, 45-73.

Poinsot, T., \& LELE, S.K. 1992 Boundary conditions for direct simulations of compressible viscous flows. J. Comp. Phys. 101, 104-129.

POPE, S. B. 1988 Evolution of surfaces in turbulence. International J. Engng. Sci. 26, 445-469.

POPE, S. B. 1990 Computations of turbulent combustion: progress and challenges. Twenty-Third Symp. (International) on Combust. 591-612. The Combustion Institute.

Rutland, C. J., \& Trouvé, A. 1991 Direct simulations of premixed turbulent flames with non-unity Lewis numbers. Combust. Flame (submitted for publication).

Trouvé, A. 1991 Simulation of flame-turbulence interactions in premixed combustion. in Annual Research Briefs - 1991, Center for Turbulence Research, Stanford Univ./NASA Ames.

Trouvé, A., \& PoInsot, T. 1992 The evolution equation for the flame surface density in turbulent premixed combustion. In Proc. 1992 Summer Program, Center for Turbulence Research, Stanford Univ./NASA Ames, 325-343.

Williams, F. A. 1985 Combustion theory. 2nd ed., Benjamin Cummings.

WRAY, A. A. 1990 Minimal storage time-advancement schemes for spectral methods. Internal Report, NASA-Ames.

Wu, M. S., Kwon, S., Driscoll, J. F., \& FAETh, G. M. 1990 Turbulent premixed hydrogen/air flames at high Reynolds numbers. Combust. Sci. Technol. 73, 327-350. 
$\longrightarrow$ 